\title{
Maternal history of child abuse moderates the association between daily stress and diurnal cortisol in pregnancy: A pilot study
}

\author{
Margaret H. Bublitz, PhD and Laura R. Stroud, PhD \\ Alpert Brown Medical School Department of Psychiatry and Human Behavior The Miriam Hospital \\ Centers for Behavioral and Preventive Medicine
}

\begin{abstract}
Previous research on the association between maternal daily stress and cortisol in pregnancy has yielded inconsistent findings. However, past studies have not considered whether stressful experiences in childhood impact maternal cortisol regulation in pregnancy. In this pilot study we aimed to examine whether the association between maternal daily stress and cortisol differed according to maternal history of child abuse. Forty-one women provided salivary cortisol samples at wake-up, 30 minutes after wake-up, and bedtime for 3 days at 3 times over second and third trimesters of pregnancy. On each day of cortisol collection women reported their daily stress. Women reported child abuse experiences prior to age 18 by completing 15 items from the Adverse Childhood Experiences scale. Twenty-one percent $(\mathrm{N}=9)$ of women reported a history of child sexual abuse (CSA), 44\% ( $\mathrm{N}=18)$ reported a history of non-sexual child abuse, and 34\% ( $\mathrm{N}=14)$ reported no history of child abuse. Hierarchical Linear Modeling (HLM) analyses revealed that stress in the day prior was associated with increases in morning cortisol in women with CSA histories compared to women with non-sexual abuse histories or no history of child abuse. Increases in evening cortisol were associated with increases in daily stress in women with CSA histories compared to women with non-sexual abuse histories or no history of child abuse. Results reveal a dynamic association between daily stress and cortisol in pregnancy and suggest that patterns differ according to maternal child abuse history.
\end{abstract}

\section{Keywords}

Daily stress; pregnancy; cortisol; child sexual abuse; diurnal; child maltreatment

\begin{abstract}
Maternal hypothalamic-pituitary-adrenal (HPA) functioning is hypothesized to act as a key mechanism linking maternal stress in pregnancy to adverse neonatal outcomes (RichEdwards et al. 2005; Wadhwa et al. 2011). However, studies on the association between stress and HPA activity in pregnancy have yielded inconsistent findings (Davis et al. 2007; Goedhart et al. 2010; Harville et al. 2009; Obel et al. 2005). Inconsistencies may be due, in part, because previous research has failed to consider whether stressful experiences in
\end{abstract}

Corresponding Author: Dr. Margaret Bublitz, 1 Hoppin Street Coro West, Providence, RI 02903 mbublitz@ lifespan.org Phone: 401-793-8086 Fax: 401-793-8056.

The authors have no conflict of interest to declare. 
childhood influence maternal HPA activity in pregnancy. This gap in the knowledgebase is significant given the robust literature showing that stressful early life experiences have profound and permanent effects on HPA regulation (Miller et al. 2010).

In this study we assessed whether associations between maternal daily stress and diurnal cortisol varied according to maternal child abuse history. Specifically, childhood sexual abuse (CSA) has been associated with both hyper and hypo-activity of the HPA axis in nonpregnant samples (Bremner et al. 2003; Cicchetti et al. 2010; De Bellis et al. 1994; Lemieux et al. 1995), and recent work by our group was the first to report that cortisol trajectories over pregnancy differ according to maternal history of CSA (Bublitz et al. 2012); women with CSA histories displayed increasing cortisol awakening responses over gestation compared to women with non-sexual abuse histories or no abuse history.

As a next step, we sought to understand whether associations between maternal daily stress and cortisol varied as a function of child abuse history. We examined whether the associations among stress and cortisol on the same day, and stress and cortisol across days, differed according to maternal child abuse history. We examined day-to-day associations between stress and cortisol because past research in non-pregnant samples shows a dynamic association between daily experiences and cortisol, such that cortisol patterns are modified both by same-day and prior-day challenge (Adam et al. 2006; Doane et al. 2010).

\section{Methods}

Participants

Women in this pilot study were pregnant women who participated in a larger study on the effects of maternal mood on fetal and infant development (Behavior and Mood in Mothers, Behavior in Infants (BAMBI) Study). Women were excluded from participating if they had medical conditions or were taking medications that could affect cortisol, reported more than one alcoholic drink per week over pregnancy, were over 40 years old, were pregnant with more than one fetus, or were at increased risk for adverse neonatal outcomes.

Forty-one pregnant women participated in the pilot study $\left(\mathrm{M}_{\mathrm{age}}=26, \mathrm{SD}=5\right)$. Women were from diverse economic (45\% of sample reported a total household income of < $\$ 30 \mathrm{~K} / \mathrm{year}$; $69 \%$ with less than a college degree) and racial (63\% Caucasian) backgrounds. Fifty-four percent of pregnancies were reported as unplanned and $55 \%$ of women reported that they were unmarried at the time of this pregnancy. This study was approved by the Women and Infants Hospital IRB.

\section{Procedure}

Women completed three study sessions at $20(\mathrm{SD}=2), 28(\mathrm{SD}=1)$, and $35(\mathrm{SD}=1)$ weeks gestation. At baseline, women completed a self-report measure of child abuse experiences, provided information on demographics, medical conditions, medications, health behaviors, mood, and information on past pregnancies. For 3 days after each of the three study sessions women completed a measure of daily stress and provided saliva cortisol samples (passive drool) at wake-up, 30 minutes after waking, and bedtime for a total of 9 days of stress/ 
cortisol collection per participant. Time of samples was verified via Medication Event Monitoring System (MEMS) caps (AARDEX, Zurich, Switzerland) for 67\% of the sample.

Child abuse history-Women were asked to report on child abuse experiences by completing 15 items from the Adverse Childhood Experiences Scale (Dube et al. 2003). This measure asked whether participants had experienced childhood sexual abuse, physical abuse, witnessed domestic violence, or experienced physical neglect prior to age 18 . Response options ranged from $0=$ never to $4=$ very often. Women who reported scores of 1 or higher were considered to have experienced abuse. Women were categorized as 'No Child Abuse (NA)' if they had never experienced any of the items in the measure, 'Non-sexual Child Abuse (CA)' if they endorsed one or more adverse experiences other than sexual abuse, and 'Child Sexual abuse (CSA)' if they endorsed having experienced child sexual abuse with or without other adverse childhood experiences.

Maternal salivary cortisol-Saliva samples were frozen at $-80^{\circ} \mathrm{C}$ and shipped to Dresden University for analysis. Cortisol concentrations were analyzed in duplicate with an immunoassay with time-resolved fluorescence detection. Intra and inter-assay coefficients of variation were $<8 \%$. For women with both self-reported and MEMS-recorded saliva sampling times there was an average time discrepancy of 5 minutes $(\mathrm{SD}=5)$ for cortisol samples at awakening, 5 minutes $(\mathrm{SD}=4)$ for cortisol samples collected 30 minutes after awakening, and 7 minutes $(\mathrm{SD}=29)$ for cortisol samples collected at bedtime. These discrepancies suggest that the majority of participants were completing cortisol samples at the self-reported sampling times. Sampling time was included as a covariate in HLM analyses.

Daily Stress-On each evening following salivary cortisol collection, women reported the severity of daily stressors by completing a modified version of the Pregnancy Experiences Scale (DiPietro et al. 2008). Women were asked, "How stressful were the following things for you today?" and were provided with a list of 13 stressors, including difficulties with their romantic partner, family, friends, financial and work stressors, thoughts about the health/ well-being of the baby, and pregnancy discomforts (i.e., sleep problems, pain). Responses ranged from $0=$ not at all to $3=$ very. Responses were summed on each day to create a daily stress variable.

Maternal Demographic Characteristics-Women provided information on demographics (age, race, marital status, income) and past births (gravida). Participants reported their pre-pregnancy height and weight to compute body mass index (BMI). Participants also provided information on mood (Inventory for Depressive Symptomatology; (Rush et al. 1986)), and anxiety (Hamilton Anxiety Rating Scale (Hamilton 1959)). At all study sessions, alcohol and cigarette consumption were measured using Timeline FollowBack interview (Sobell et al. 1996). Participants in this study were not included in our previous study on maternal child abuse history and cortisol awakening responses over gestation (Bublitz \& Stroud 2012). 


\section{Data Analysis}

Salivary cortisol values were log transformed to adjust for skewed distributions. In order to accurately capture the cortisol awakening response, morning saliva samples that were collected $<20$ or $>40$ minutes apart were removed from analyses. This resulted in missing values for $10 \%$ of cortisol samples at awakening (355 samples out of a possible 369 for analysis of stress and cortisol on the same day; 237 samples out of a possible 246 samples for analysis of stress and cortisol the next day) and 10\% of samples collected 30 minutes after awakening (352 samples out of a possible 369 for analysis of stress and cortisol on the same day; 236 samples out of a possible 246 samples for analysis of stress and cortisol the next day). As well, $9 \%$ of evening cortisol samples were missing due to non-completion or non-compliance (348 samples out of a possible 348 for analysis of stress and cortisol on the same day; 236 samples out of 246 possible samples for analysis of stress and cortisol the next day).

To determine whether associations between daily stress and cortisol were moderated by maternal child abuse group, we computed two-level hierarchical linear models (HLM) using Hierarchical Linear Modeling software (Raudenbush et al. 2002). At Level-1 we examined the within-person associations among daily stress and cortisol. At level-2 models we examined whether associations among daily stress and cortisol were moderated by maternal child abuse group (and significant covariates). Models were computed separately for cortisol values at awakening, 30 minutes following awakening, and bedtime. Time of sampling and gestational age at sampling were included as covariates in all models.

We computed three models to examine same-day associations between stress and cortisol (trajectories over awakening, 30 minutes after awakening, and evening) and three models to examine associations between prior-day stress and cortisol (trajectories over awakening, 30 minutes after awakening, and evening). In models in which we examined same-day associations between cortisol and stress, stress was considered the dependent variable given that women reported their daily stress after providing saliva samples. In models in which we examined associations between prior-day stress and cortisol, cortisol was modeled as the dependent variable because samples were collected after the experience of stress. This analytic approach has been employed in prior studies (Adam 2006; Doane \& Adam 2010). Analyses of same-day associations between stress and cortisol included all 9 days of data collection over pregnancy. Analyses of associations among daily stress and next-day cortisol included 6 days of data over pregnancy as we did not have cortisol data on the day after the last day of women reported on daily stress.

If maternal history of child abuse significantly moderated associations between daily stress and cortisol we performed post-hoc HLM models to test whether the relationship between daily stress and cortisol differed between pairs of child abuse groups (i.e., CSA vs no abuse). In figures 1 and 2 we used HLM software to model the predicted patterns of associations between daily stress and cortisol among women with CSA histories, non-sexual, or no abuse histories. Daily stress and cortisol were assessed as continuous variables; however, HLM software plots the associations among stress and cortisol at the $25^{\text {th }}$ and $75^{\text {th }}$ percentiles of the distribution to model the within-person predicted patterns of associations. While log 
transformed cortisol values were included in analyses, raw values are presented in the figures. Figures contain brackets to indicate significant differences in paired comparisons of maternal child abuse groups.

\section{Results}

\section{Descriptive Statistics}

Twenty-one percent ( $\mathrm{N}=9$ ) of women reported a history of child sexual abuse (CSA), $44 \%$ $(\mathrm{N}=18)$ reported a history of non-sexual child abuse, and $34 \%(\mathrm{~N}=14)$ reported no history of child abuse. One-way analyses of variance (ANOVA) and chi-square tests were performed using SPSS software for Windows (version 19) to assess whether child abuse groups differed on maternal characteristics. Child abuse groups significantly differed only on gravida $(F(2,18)=4.22, p=.03)$. Tukey's post hoc comparisons showed that women with a history of CSA reported a greater number of past pregnancies than NA women ( $p=.01)$, and non-CSA women reported a marginally greater number of past pregnancies than NA women $(p=.10)$. Gravida was included as a covariate in analyses. No other differences were found among child abuse groups.

We also examined associations between maternal characteristics, cortisol and stress using hierarchical linear models. Results showed that maternal income significantly predicted cortisol at awakening $(\mathrm{b}=.70, \mathrm{SE}=.30, p=.03)$, but not 30 minutes after waking $(\mathrm{b}=.69, \mathrm{SE}=$. $53, p=.20)$ or bedtime ( $\mathrm{b}=-.14, \mathrm{SE}=.19, p=.48)$. As income increased, maternal cortisol at awakening also increased. Maternal income was included as a covariate in all models. No other significant associations were found between maternal characteristics and cortisol levels at awakening ( $p$ 's >.14), cortisol levels 30 minutes after awakening ( $p$ 's > .13) or bedtime cortisol levels $(p$ 's $>.16)$.

Analyses of associations between maternal characteristics and daily stress revealed that, as gravida increased, maternal daily stress increased $(\mathrm{b}=1.70, \mathrm{SE}=.60, p=.01)$. As previously noted, gravida was included as a covariate in all models. In addition, as symptoms of depression increased daily stress increased $(\mathrm{b}=.43, \mathrm{SE}=.14, p=.005)$. However, because depressive symptoms did not predict cortisol, depressive symptoms were not included as a covariate in subsequent models. No other significant associations were found between daily stress and maternal characteristics $(p ' s>.12)$.

All women delivered babies that were full-term $(\mathrm{M}=39.69$ weeks gestation; range: 38.13-41.86). No babies were born low birth weight ( $\mathrm{M}=3,336$ grams; range: 2,605-4,610). APGAR scores, a measure of the physical condition of a newborn infant rated on a scale from 0-10 with higher scores indicating better health, reflected that babies in this sample were in good health when babies were scored 5 minutes following delivery $(M=9$; range: 7-10). Neonatal outcomes did not significantly differ according to maternal child abuse group $(p ' s>.15)$.

\section{Associations between daily stress and diurnal cortisol by child abuse group}

All results are presented in Table 1. Results from HLM analyses examining maternal abuse as a moderator of the association between daily stress and same-day cortisol revealed that 
abuse history significantly moderated associations between evening cortisol levels and stress reported the same evening $(\mathrm{b}=.14, \mathrm{SE}=.07, p=.04)$. See Figure 1. Post hoc paired-group comparisons revealed that, as evening cortisol levels increased, self-reported stress levels were higher in women with child sexual abuse histories vs. women with no abuse history $(\mathrm{b}=.13, \mathrm{SE}=.06, p=.05)$. As well, evening cortisol levels increased stress levels were marginally higher in women with child sexual abuse histories versus non-sexual abuse histories levels ( $\mathrm{b}=.24, \mathrm{SE}=.13, p=.07$ ). Finally, as evening cortisol levels increased there were no significant differences in daily stress levels in women with non-sexual abuse histories vs. women with no abuse history ( $\mathrm{b}=.01, \mathrm{SE}=.05, p=.75$ ). Maternal child abuse history did not significantly moderate associations among morning cortisol levels and stress reported the same evening $(p>.45)$.

Next we examined whether maternal history of child abuse moderated the associations among prior-day stress and cortisol. Results revealed that maternal history of child abuse significantly moderated the association between prior-day stress and cortisol at 30 minutes post awakening ( $\mathrm{b}=.79, \mathrm{SE}=.33, p=.02$ ). See Figure 2. Post hoc analyses of group differences revealed that, as prior-day stress increased, cortisol at 30 minutes after awakening increased in women with CSA histories vs. women with no history of child abuse $(\mathrm{b}=.69, \mathrm{SE}=.32, p=.01)$. Cortisol levels at 30 minutes after awakening increased as prior-day stress increased in women with non-sexual child abuse histories vs. women with no abuse $(b=3.67, \mathrm{SE}=.99, p=.001)$. Finally, post hoc analyses revealed that as prior-day stress increased, cortisol levels 30 minutes after awakening increased in women with sexual abuse histories compared to women with child non-sexual abuse histories ( $\mathrm{b}=-2.67, \mathrm{SE}=.78, p=$. 002). Maternal history of child abuse did not significantly moderate the association between daily stress and next-day cortisol at awakening or at bedtime $(p>.30)$.

\section{Discussion}

The aim of this pilot study was to assess whether the associations between daily stress and cortisol in pregnancy varied according to maternal history of child abuse. We found that increases in evening cortisol were associated with increases in daily stress in women with CSA histories compared to women with non-sexual abuse histories or no history of child abuse. As well, we found that prior-day stress was associated with increases in morning cortisol in women with CSA histories compared to women with non-sexual abuse histories or no history of child abuse. These results are consistent with those from past studies in nonpregnant samples showing that prior-day feelings of loneliness and sadness were associated with altered diurnal cortisol rhythms, including greater morning cortisol output and flatter cortisol slopes (Adam et al. 2006; Doane \& Adam 2010). According to the "boost" hypothesis proposed by Adam et al. (2006), an increase in morning cortisol following adverse prior-day experiences may provide the individual with extra energy and resources to help manage future daily challenges. As an alternative explanation, Adam et al. (2006) also suggested that higher morning cortisol levels could reflect a (maladaptive) prolonged stress response that carried over from the previous day. Future research is needed to examine potential consequences of these altered cortisol patterns on maternal and neonatal health. 
Previous studies have shown that, over typically developing pregnancies, morning cortisol levels decline as pregnancy progresses (Buss et al. 2009; Entringer et al. 2010). Thus, cortisol patterns observed in this study may be indicative of maternal HPA dysregulation. Results also indicate that women with CSA histories may display greater HPA dysregulation following stress compared to women with histories of child abuse that was non-sexual in nature. Prior findings on the impact of child maltreatment type on cortisol regulation are mixed (Bremner et al. 2003; Cicchetti et al. 2010; De Bellis et al. 1994; Lemieux \& Coe 1995); however, in pregnancy, prior work by our group found that women with CSA histories displayed more dysregulated diurnal cortisol patterns compared to women with non-sexual child abuse histories and no abuse history (Bublitz \& Stroud 2012). As well, past research has found that women with CSA histories are at greater risk for pregnancy complications compared to women with other forms of abuse (Leeners et al. 2010; Noll et al. 2007). Thus, maternal cortisol dysregulation following stress may serve as a biological pathway linking CSA history to adverse neonatal outcomes, though future studies are needed to examine this hypothesis.

This pilot study was the first, to our knowledge, to examine whether the association between maternal daily stress and cortisol in pregnancy differed according to maternal history of child abuse. Results are preliminary and limited by the small sample size and retrospective recall of maternal history of child abuse. As well, we included a small number of daily saliva samples in order to minimize participant burden; however this may limit the accuracy of our measure of diurnal cortisol rhythms. Despite these limitations, findings shed light on the dynamic association between daily experiences and diurnal cortisol in pregnancy, and the importance of considering associations both same-day and prior-day challenge when examining maternal cortisol output over pregnancy.

\section{Acknowledgments}

Funding for this study was provided by NIH grant R01MH079153 to LRS. MHB's time was supported by T32HL076134-07.

\section{References}

Adam EK. Transactions among adolescent trait and state emotion and diurnal and momentary cortisol activity in naturalistic settings. Psychoneuroendocrinology. 2006; 31(5):664-79. [PubMed: 16584847]

Adam EK, Hawkley LC, Kudielka BM, et al. Day-to-day dynamics of experience--cortisol associations in a population-based sample of older adults. Proc Natl Acad Sci U S A. 2006; 103(45): 17058-63. [PubMed: 17075058]

Bremner JD, Vythilingam M, Anderson G, et al. Assessment of the hypothalamic-pituitary-adrenal axis over a 24-hour diurnal period and in response to neuroendocrine challenges in women with and without childhood sexual abuse and posttraumatic stress disorder. Biological Psychiatry. 2003; 54:710-8. [PubMed: 14512211]

Bublitz MH, Stroud LR. Childhood sexual abuse predicts diurnal cortisol in pregnancy: preliminary findings. Psychoneuroendocrinology. 2012

Buss C, Entringer S, Reyes JF, et al. The maternal cortisol awakening response in human pregnancy is associated with the length of gestation. Am J Obstet Gynecol. 2009; 201(4):398, e1-8. [PubMed: 19716542] 
Cicchetti D, Rogosch FA, Gunnar MR, et al. The Differential Impacts of Early Physical and Sexual Abuse and Internalizing Problems on Daytime Cortisol Rhythm in School-Aged Children. Child Development. 2010; 81(1):252-69. [PubMed: 20331666]

Davis EP, Glynn L, Dunkel-Schetter C, et al. Prenatal exposure to maternal depression and cortisol influences infant temperament. Journal of the American Academy of Child and Adolescent Psychiatry. 2007; 46(6):737-46. [PubMed: 17513986]

De Bellis MD, Chrousos GP, Dorn LD, et al. Hypothalamic-pituitary-adrenal axis dysregulation in sexually abused girls. The Journal of Clinical Endocrinology and Metabolism. 1994; 78:249-55. [PubMed: 8106608]

DiPietro JA, Christensen AL, Costigan KA. The Pregnancy Experiences Scale - Brief Version. J Psychosom Obstet Gynaecol. 2008; 29(4):262-7. [PubMed: 19065395]

Doane LD, Adam EK. Loneliness and cortisol: momentary, day-to-day, and trait associations. Psychoneuroendocrinology. 2010; 35(3):430-41. [PubMed: 19744794]

Dube SR, Felitti VJ, Dong M, et al. Childhood abuse, neglect, and household dysfunction and the risk of illicit drug use: the adverse childhood experiences study. Pediatrics. 2003; 111(3):564-72. [PubMed: 12612237]

Entringer S, Buss C, Shirtcliff EA, et al. Attenuation of maternal psychophysiological stress responses and the maternal cortisol awakening response over the course of human pregnancy. Stress. 2010; 13(3):258-68. [PubMed: 20067400]

Goedhart G, Vrijkotte TG, Roseboom TJ, et al. Maternal cortisol and offspring birthweight: results from a large prospective cohort study. Psychoneuroendocrinology. 2010; 35(5):644-52. [PubMed: 19889503]

Hamilton M. The assessment of anxiety states by rating. Br J Med Psychol. 1959; 32(1):50-5. [PubMed: 13638508]

Harville EW, Savitz DA, Dole N, et al. Stress Questionnaires and Stress Biomarkers during Pregnancy. Journal of Women's Health. 2009; 18(9):1425-33.

Leeners B, Stiller R, Block E, et al. Pregnancy complications in women with childhood sexual abuse experiences. Journal of Psychosomatic Research. 2010; 69:503-10. [PubMed: 20955870]

Lemieux AM, Coe CL. Abuse-related posttraumatic stress disorder: Evidence for chronic neuroendocrine activation in women. Psychosomatic Medicine. 1995; 57:105-15. [PubMed: 7792368]

Miller GE, Chen E. Harsh family climate in early life presages the emergence of a proinflammatory phenotype in adolescence. Psychological Science. 2010; 21(6):848-56. [PubMed: 20431047]

Noll J, Schulkin J, Trickett PK, et al. Differential pathways to preterm delivery for sexually abused and comparison women. Journal of Pediatric Psychology. 2007; 32(10):1238-48. [PubMed: 17569710]

Obel C, Hedegaard M, Henriksen TB, et al. Stress and salivary cortisol during pregnancy. Psychoneuroendocrinology. 2005; 30:647-56. [PubMed: 15854781]

Raudenbush, SW.; Bryk, AS., editors. Hierarchical linear models: applications and data analysis methods. 2 edn.. SAGE; 2002. p. 485

Rich-Edwards JW, Grizzard TA. Psychosocial stress and neuroendocrine mechanisms in preterm delivery. American Journal of Obstetrics and Gynecology. 2005; 192(5 Suppl):S30-5. [PubMed: 15891710]

Rush AJ, Giles DE, Schlesser MA, et al. The Inventory for Depressive Symptomatology (IDS): preliminary findings. Psychiatry Res. 1986; 18(1):65-87. [PubMed: 3737788]

Sobell IC, Brown J, Leo GI, et al. The reliability of the Alcohol Timeline Followback when administered by telephone and by computer. Drug Alcohol Depend. 1996; 42:49-54. [PubMed: 8889403]

Wadhwa PD, Entringer S, Buss C, et al. The contribution of maternal stress to preterm birth: Issues and considerations. Clin Perinatol. 2011; 38:351-84. [PubMed: 21890014] 
—Child Sexual Abuse - - Non-sexual Abuse $\cdots . .$. No Abuse

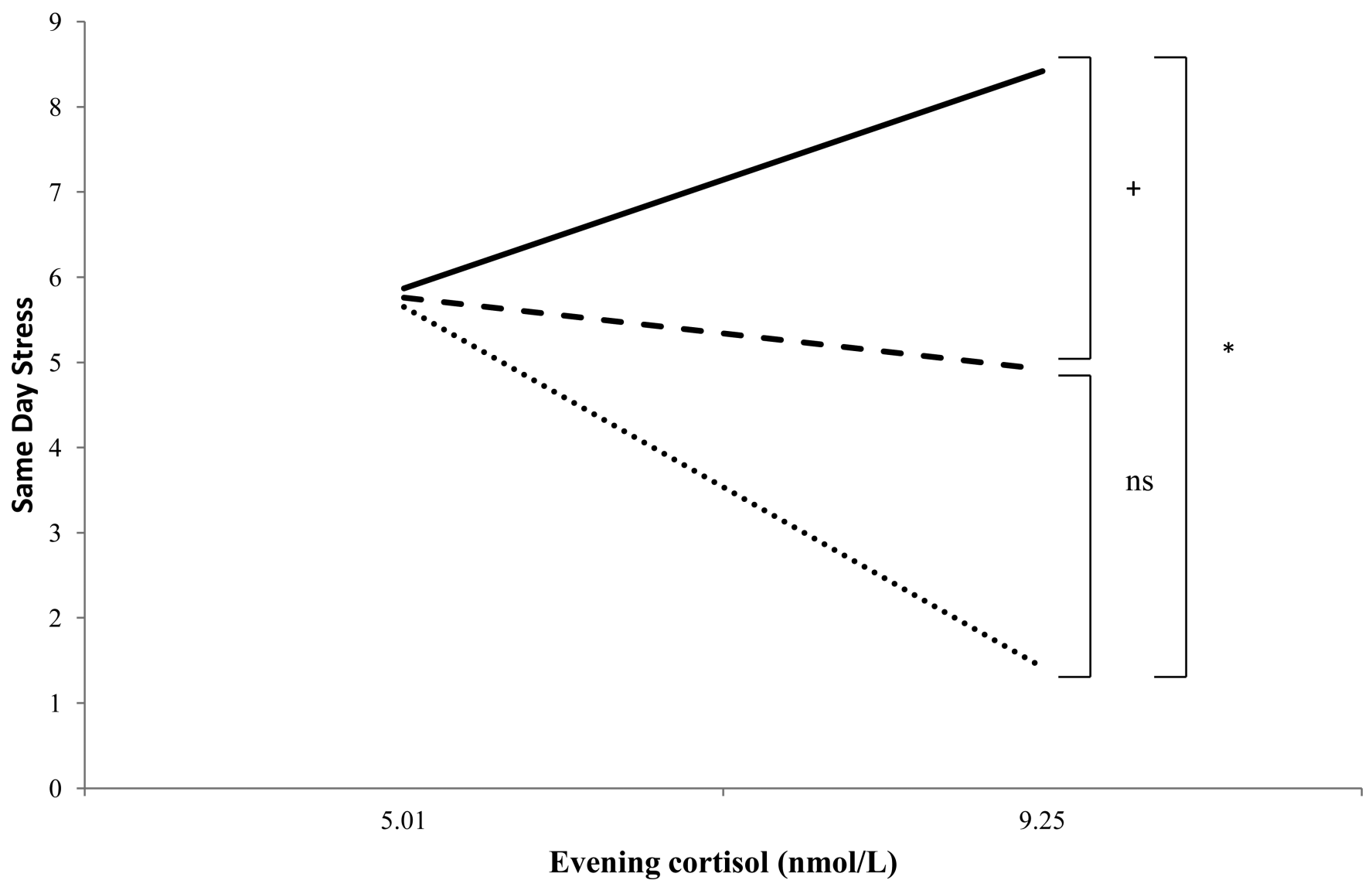

Figure 1.

Associations between evening cortisol and same-day stress by maternal child abuse group. Note. Brackets indicate results from post hoc paired group comparisons of child abuse groups. Hierarchical linear models controlled for time of sampling, gestational age at assessment, maternal income, and gravida.

$+p<.10 ; * p<.05 ; \mathrm{ns}=$ not significant. 
—Child Sexual Abuse _ - Non-sexual Abuse $\quad \cdots \cdot$. No Abuse

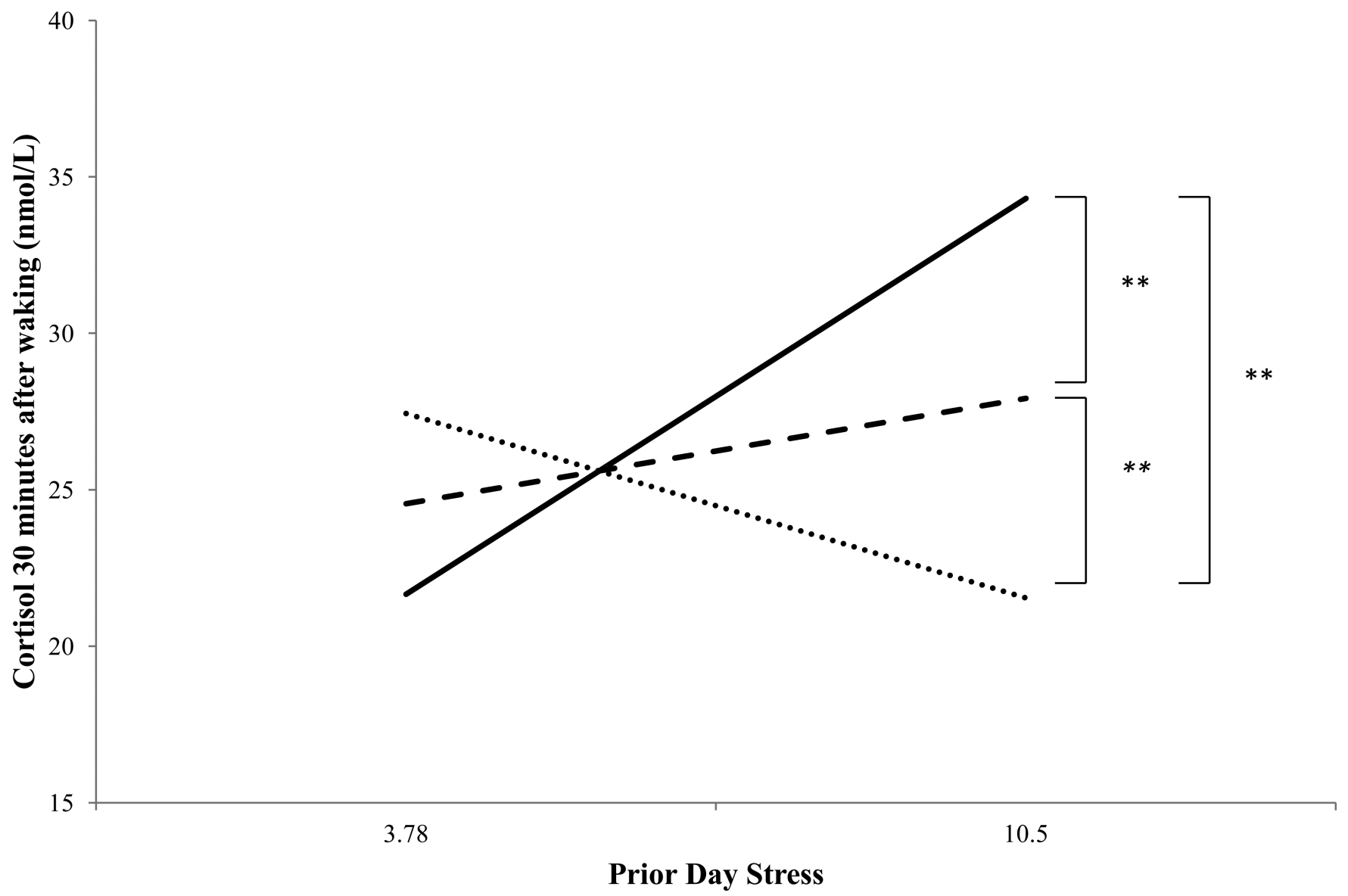

Figure 2.

Associations between prior-day stress and cortisol 30 minutes after waking by maternal child abuse group.

Note. Brackets indicate results from post hoc paired group comparisons of child abuse groups. Hierarchical linear models controlled for time of sampling, gestational age at assessment, maternal income, and gravida.

$* * p<.01$ 
Table 1

Maternal and Infant Characteristics by Maternal Child Abuse Group.

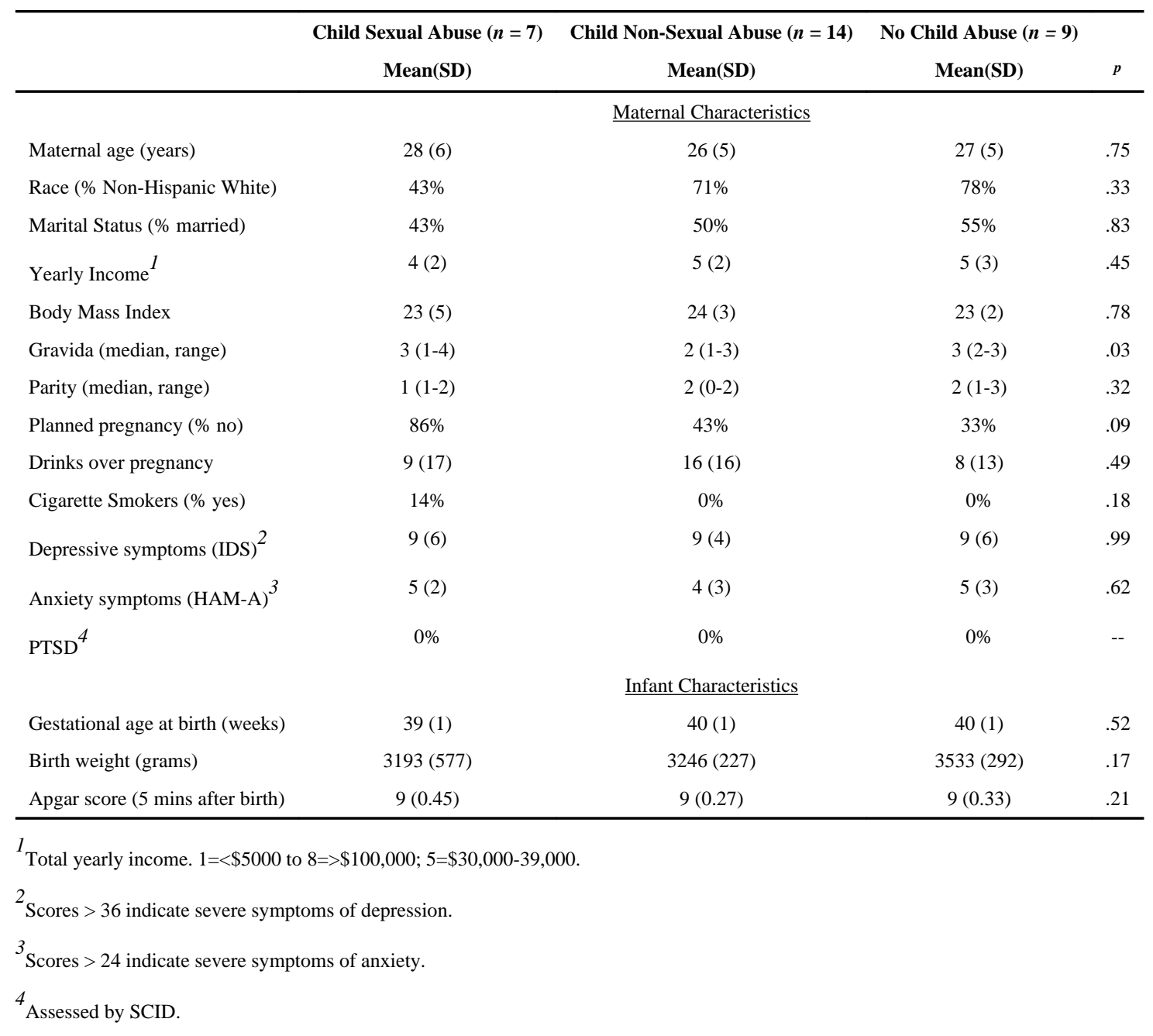

